\title{
Avaliação da satisfação dos pacientes e familiares em um serviço de saúde mental na cidade de Rio Branco, Acre
}

\author{
Evaluation of satisfaction of patients and relatives in \\ a mental health service in the city of Rio Branco, Acre
}

Priscila Oliveira de Mirandaํ', Orivaldo Florêncio de Souza', Terezinha de Freitas Ferreira ${ }^{1}$

\section{RESUMO}

Objetivo: Avaliar a satisfação dos pacientes e familiares atendidos em um serviço ambulatorial de saúde mental da cidade de Rio Branco, Acre. Métodos: Foi realizado um estudo transversal com uma amostra de 160 pacientes e 160 familiares. Para coleta de dados, foram utilizadas as versões abreviadas das Escalas de Satisfação com os Serviços de Saúde Mental - SATIS-BR para pacientes e familiares, e um questionário sociodemográfico e clínico. Foram feitos análises estatísticas descritivas, cálculos das médias e desvios-padrão dos escores de satisfação global e das subescalas, e análises bivariadas utilizando o programa SPSS, versão 17. Resultados: Os resultados da média de satisfação global dos pacientes e familiares revelaram que eles estão satisfeitos com o serviço de saúde mental. As subescalas dos pacientes: competência e compreensão da equipe e acolhida da equipe e ajuda recebida foram elevadas. No entanto, a subescala condições físicas e conforto do serviço apresentou uma menor média de satisfação. Também apresentaram um elevado nível de satisfação as subescalas para os familiares: resultados do tratamento, acolhida e competência da equipe e privacidade

\section{Palavras-chave}

Avaliação de serviços de saúde, satisfação do paciente, serviços de saúde mental, cuidadores. e confidencialidade. Foi identificado que pacientes mais velhos e que não tinham tido crises estavam mais satisfeitos. Assim como os familiares mais jovens também tinham maior nível de satisfação. Conclusão: Os resultados apontam para necessidades de melhorias nos aspectos relacionados a infraestrutura, conforto e aparência dos serviços, bem como a criação de estratégias que favoreçam maior participação do familiar no tratamento do paciente.

\section{ABSTRACT}

Objective: Evaluate the satisfaction of patients and family members attended an outpatient mental health service of the city of Rio Branco, Acre. Methods: It was conducted a sectional study with a sample of 160 patients and 160 family members. To collect data, it was used the abbreviated versions of the Scales of Satisfaction with Mental Health Services - SATIS-BR for patients and relatives, and sociodemographic and clinical questionnaire. It was performed descriptive statistical analyzes, calculations of averages and standard scores for overall satisfaction and subscales deviations, and bivariate analyzes using SPSS, version 17. Results: The

1 Universidade Federal do Acre (UFAC), Programa de Pós-graduação em Saúde Coletiva.

$$
\begin{gathered}
\text { Recebido em } \\
\text { 26/6/2014 } \\
\text { Aprovado em } \\
\text { 8/12/2014 }
\end{gathered}
$$

DOI: 10.1590/0047-2085000000042
Endereço para correspondência: Priscila Oliveira de Miranda

Rua João Nascimento, 70, Conjunto Adalberto Sena

69921-162 - Rio Branco, Acre, Brasil

E-mail: priscilamiranda18@yahoo.com.br 


\section{Keywords}

Evaluation of health

services, patient

satisfaction, mental health

services, caregivers. results of average overall patient satisfaction and relatives revealed that they are satisfied with the mental health service. The subscales of patients: competence and understanding of staff, and acceptance from the team and received help were high; however, the subscale physical conditions and service comfort had a lower average satisfaction. They also showed a high level of satisfaction subscales for the relatives: results of treatment, acceptance and competence of staff and privacy and confidentiality. It was identified that older patients and the who had no seizures were more satisfied, as much as the younger relatives also had a higher level of satisfaction. Conclusion: The results indicate the need for improvements in aspects of infrastructure, comfort and appearance of the services, and the creation of strategies that facilitate greater participation of relatives in the patient care.

\section{INTRODUÇÃO}

As conquistas advindas da reforma psiquiátrica implicaram tanto a construção como a implantação de serviços substitutivos em oposição ao modelo manicomial. Esses serviços devem ser baseados na inclusão dos pacientes e no tratamento multidisciplinar, garantindo a utilização de múltiplos recursos e resolutividade. Dessa forma, a Organização Mundial de Saúde (OMS) tem destacado a importância da avaliação e do monitoramento da qualidade dos serviços de saúde mental, objetivando verificar a eficácia dos programas de prevenção e de tratamento de saúde mental'.

Diante do novo contexto de saúde mental, os pacientes passaram a participar ativamente do seu tratamento, além de suas avaliações subjetivas serem consideradas como complemento dos resultados clínicos obtidos². Assim, a avaliação dos serviços de saúde mental por meio da perspectiva do usuário torna-se um desafio indispensável para a construção de espaços onde o indivíduo tenha possibilidade de exercer sua cidadania, com liberdade de expressão, e que, principalmente, se reconheça como agente de mudança ${ }^{3}$.

Considerando o paciente apto a julgar o serviço que the foi prestado e mensurar o quanto isso está interferindo no seu bem-estar, as instituições devem valorizar esses julgamentos nas suas tomadas de decisões, uma vez que essa valoração poderá contribuir para o direcionamento de novas estratégias ou melhorias das existentes a fim de garantir a qualidade do serviço prestado ${ }^{4}$.

A avaliação da satisfação dos familiares de pacientes que utilizam os serviços de saúde mental também é igualmente importante. Com a desinstitucionalização, os familiares são as pessoas mais próximas do paciente, que acompanham a rotina, supervisionam o tratamento, lidam com os comportamentos problemáticos e avaliam as mudanças que ocorrem. Dessa forma, no papel de principais cuidadores possuem informações relevantes para auxiliar os profissionais na tomada de decisões em relação ao tratamento oferecido5.

No intuito de avaliar a qualidade dos serviços de saúde mental, considerando a percepção de pacientes e familiares, têm sido bastante utilizados como instrumento os estudos de satisfação, haja vista que estes proporcionam uma visão ampliada de quais fatores do serviço devem ser melhorados ${ }^{6}$.

Apesar das recomendações sobre a necessidade de avaliação contínua dos serviços de saúde mental, na Região Norte do País, até o momento, não foi encontrada nenhuma pesquisa que tenha estudado a satisfação com os serviços de saúde mental. Visando contribuir para suprir essa lacuna, este estudo teve como objetivo avaliar o grau de satisfação dos pacientes e familiares em um ambulatório de saúde mental na cidade de Rio Branco, Acre.

\section{MÉTODOS}

\section{Delineamento do estudo}

Este é um estudo transversal, realizado de outubro de 2012 a janeiro de 2013, com aplicação de escalas de medida multifatorial de satisfação dos usuários e familiares.

\section{Participantes}

Participaram deste estudo pacientes e os familiares cuidadores dos pacientes. Os pacientes recebiam tratamento do tipo ambulatorial em uma instituição pública de saúde mental na cidade de Rio Branco, Acre. O tamanho da amostra de pacientes e familiares foi definido adotando poder de amostra $(1-\beta)=0,80$, nível de significância $a=0,05$, e tamanho do efeito $\Delta=0,25$. Utilizando esses valores, chegou-se a um tamanho de amostra igual a 128 pacientes e 128 familiares segundo tabelas de Machin et al.? . A esse valor foram acrescidos $25 \%$ para correção de recusas, totalizando 160 pacientes e 160 familiares entrevistados.

Foram incluídos na amostra os pacientes maiores de 18 anos, os quais estavam recebendo tratamento ambulatorial há, no mínimo, um ano. Foram excluídos os pacientes que estavam internados ou em crise e aqueles que demonstraram impossibilidade em compreender e responder às questões dos instrumentos utilizados.

Para cada paciente selecionado, foi identificado o seu familiar cuidador para compor a amostra de familiares. Foram incluídos os familiares maiores de 18 anos, de ambos 
os sexos e que residiam com o paciente. Foram excluídos os que não conseguiram responder aos instrumentos de avaliação, bem como os que apresentavam transtorno mental diagnosticado.

\section{Coleta de dados}

A coleta de dados foi realizada por meio de questionários aplicados individualmente pela pesquisadora e dois entrevistadores treinados. A coleta de dados com os pacientes e familiares foi realizada na instituição de segunda a sexta-feira, nos dois turnos de atendimento, até ser completado o tamanho da amostra. Todos os pacientes que chegavam à instituição para consulta ambulatorial na presença de um acompanhante foram abordados para verificar se atendiam aos critérios para participação no estudo.

Antes de iniciar a aplicação dos questionários, os entrevistadores se apresentavam, explicavam os objetivos da pesquisa, asseguravam o sigilo das informações coletadas e o anonimato de cada participante, faziam a leitura do termo de consentimento livre e esclarecido e a coleta da assinatura do documento. Este estudo foi aprovado pela direção do serviço de saúde mental e pela Comissão de Ética em Pesquisa com Seres Humanos da Universidade Federal do Acre, sob o número de CAAE 06581712.0.0000.5010.

\section{Instrumentos de medidas e variáveis}

A satisfação dos pacientes e familiares com os serviços de saúde mental foi avaliada por meio das versões abreviadas das Escalas de Avaliação da Satisfação com os Serviços de Saúde Mental - SATIS-BR. As escalas SATIS-BR integram um conjunto de escalas para avaliação de serviços de saúde mental, elaboradas pela Divisão de Saúde Mental da OMS, que foram validadas para o Brasil por Bandeira et al.89, Bandeira et al. ${ }^{10} \mathrm{e}$ Bandeira e Silva"1. As escalas são compostas de questões com alternativas de respostas dispostas em escala ordinal tipo Likert de 5 pontos, em que $1=$ muito insatisfeito, $2=$ insatisfeito, 3 = indiferente, 4 = satisfeito, e 5 = muito satisfeito.

A versão abreviada da SATIS-BR/pacientes possui 12 itens quantitativos, distribuídos em três subescalas: (1) satisfação com a competência e compreensão da equipe, (2) satisfação com a acolhida da equipe e ajuda recebida e (3) satisfação com as condições físicas e conforto do serviço, além de três questões abertas referentes à percepção dos usuários sobre diversos aspectos dos serviços recebidos.

A versão abreviada da SATIS-BR/Familiares possui oito itens quantitativos, distribuídos em três subescalas: (1) satisfação com o resultado do tratamento, (2) satisfação com a acolhida e a competência da equipe e (3) satisfação com a privacidade e confidencialidade no serviço, além de três questões referentes à percepção dos familiares sobre diversos aspectos dos serviços recebidos.

Além das escalas foi aplicado um questionário para avaliar as características sociodemográficas dos pacientes e fa- miliares e as características clínicas dos pacientes. As variáveis sociodemográficas avaliadas foram: sexo, idade, estado civil, escolaridade, situação ocupacional e renda. E as características clínicas foram: diagnóstico, idade do início do transtorno psiquiátrico, duração do transtorno e do tratamento psiquiátrico, número de crises no último ano e de internações. Os diagnósticos foram coletados do prontuário ou do boletim de atendimento diário seguindo a Classificação Estatística Internacional de Doenças e Problemas Relacionados à Saúde - CID-10, e as demais variáveis clínicas foram referidas pelos próprios pacientes.

\section{Análise dos dados}

Os dados foram inseridos em banco de dados construído no programa EpiData, versão 3.0, e as análises estatísticas foram realizadas utilizando o programa Statistical Package for Social Sciences - SPSS, versão 17.

Para a descrição das características das amostras foram calculadas as frequências absolutas e relativas para as variáveis categóricas, e as médias e seus respectivos desvios-padrão (DP) para as variáveis contínuas. Também foram calculados a média e os respectivos desvios-padrão dos escores de satisfação global e das subescalas.

$\mathrm{Na}$ análise bivariada, foram analisadas as relações entre os escores de satisfação global e as variáveis sociodemográficas e clínicas. A escolha dos testes estatísticos para a análise comparativa dos dados foi definida a partir da verificação da hipótese de normalidade. A aplicação do teste de Kolmogorov-Smirnov indicou a não normalidade dos dados das escalas (SATIS-BR/Pacientes: KS = 1,34; $p=0,05$ e SATIS-BR/ Familiares: $K S=1,83 ; p=0,00$ ), o que justificou a utilização dos testes não paramétricos. Dessa forma, para as variáveis dicotômicas foi utilizado o teste Mann-Whitney e, para as variáveis com três categorias, o teste de Kruskal-Wallis. Em todos os testes estatísticos foi utilizado, como nível de significância, o valor de $p \leq 0,05$.

\section{RESULTADOS}

Os pacientes em sua maioria eram do sexo feminino (72,5\%), viviam com companheiro $(55,6 \%)$ e a escolaridade predominante era o ensino fundamental (47,5\%). A idade média era de 41,93 anos (DP: 12,98), sendo a mínima 18 e a máxima 80 anos. Em relação à situação ocupacional, 14,4\% trabalhavam. No entanto, $68,1 \%$ possuíam renda própria, destes $54,3 \%$ recebiam de 1 a 2 salários-mínimos, e a principal fonte de renda era auxílio do governo (37,5\%), seguida de aposentadoria ou pensão (22,5\%) (Tabela 1).

A categoria diagnóstica predominante foi a de transtornos do humor e afetivos (51,9\%), conforme os critérios da CID-10. A idade de início do transtorno psiquiátrico foi, em média, de 26,34 anos (DP: 15,29) e a duração média do trans- 
torno psiquiátrico, de 15,48 anos (DP: 12,04), com amplitude de 1 a 50 anos. A duração média do tratamento psiquiátrico era de 8,77 anos (DP: 8,02), com tempo mínimo de 1 e

Tabela 1. Características sociodemográficas dos pacientes e familiares atendidos no ambulatório do Hospital de Saúde Mental do Acre, Rio Branco, Acre, 2012/2013

\begin{tabular}{|c|c|c|}
\hline Variáveis & $\begin{array}{c}\text { Pacientes } \\
\mathrm{n}(\%)\end{array}$ & $\begin{array}{c}\text { Familiares } \\
\mathrm{n}(\%)\end{array}$ \\
\hline \multicolumn{3}{|l|}{ Sexo } \\
\hline Masculino & $44(27,5)$ & $71(44,4)$ \\
\hline Feminino & $116(72,5)$ & $89(55,6)$ \\
\hline \multicolumn{3}{|l|}{ Idade } \\
\hline 18 a 29 anos & $24(15,0)$ & $25(15,6)$ \\
\hline 30 a 49 anos & $94(58,8)$ & $74(46,3)$ \\
\hline 50 anos ou mais & $42(26,2)$ & $61(38,1)$ \\
\hline \multicolumn{3}{|l|}{ Escolaridade } \\
\hline Analfabeto & $26(16,2)$ & $14(8,8)$ \\
\hline Ensino Fundamental & $76(47,5)$ & $82(51,2)$ \\
\hline Ensino Médio & $46(28,8)$ & $55(34,4)$ \\
\hline Ensino Superior & $12(7,5)$ & $9(5,6)$ \\
\hline \multicolumn{3}{|l|}{ Situação conjugal } \\
\hline Com companheiro & $89(55,6)$ & $117(73,1)$ \\
\hline Sem companheiro & $71(44,4)$ & $43(26,9)$ \\
\hline \multicolumn{3}{|l|}{ Situação ocupacional } \\
\hline Trabalha & $23(14,4)$ & $82(51,2)$ \\
\hline Não trabalha & $137(85,6)$ & $78(48,8)$ \\
\hline \multicolumn{3}{|l|}{ Possui renda própria } \\
\hline Sim & $109(68,1)$ & $123(76,9)$ \\
\hline Não & $51(31,9)$ & $37(23,1)$ \\
\hline \multicolumn{3}{|l|}{ Renda (em salários-mínimos) } \\
\hline Menor que 1 & $16(10,0)$ & $17(10,6)$ \\
\hline 1 a 2 & $87(54,3)$ & $89(55,6)$ \\
\hline 2,1 a 3 & $3(1,9)$ & $9(5,6)$ \\
\hline Maior que 3 & $3(1,9)$ & $8(5,0)$ \\
\hline Não possui renda & $51(31,9)$ & $37(23,2)$ \\
\hline \multicolumn{3}{|l|}{ Origem da renda } \\
\hline Emprego ou trabalho regular & $13(8,1)$ & $55(34,4)$ \\
\hline Aposentadoria ou pensão & $36(22,5)$ & $54(33,8)$ \\
\hline Auxílio do governo & $60(37,5)$ & $14(8,7)$ \\
\hline Não possui renda & $51(31,9)$ & $37(23,1)$ \\
\hline \multicolumn{3}{|l|}{ Parentesco com o paciente } \\
\hline Mãe ou pai & - & $30(18,7)$ \\
\hline Irmão(a) & - & $14(8,7)$ \\
\hline Cônjuge & - & $70(43,8)$ \\
\hline Filho & - & $35(21,9)$ \\
\hline Outro & - & $11(6,9)$ \\
\hline
\end{tabular}

máximo de 45 anos. Quanto ao número de crises no último ano, 58,7\% dos pacientes tinham sofrido pelo menos uma crise. Dos pacientes estudados, 41,3\% relataram que haviam sido internados em hospitais psiquiátricos, em média, 1,49 vez (DP: 3,61) no decorrer da vida, com amplitude de 1 a 31 internações (Tabela 2).

Tabela 2. Características clínicas dos pacientes atendidos no ambulatório do Hospital de Saúde Mental do Acre, Rio Branco, Acre, 2012/2013

\begin{tabular}{|c|c|c|}
\hline Variáveis & $\mathrm{n}(\%)$ & Média (DP) \\
\hline \multicolumn{3}{|l|}{ Categoria diagnóstica } \\
\hline $\begin{array}{l}\text { Esquizofrenia, } \\
\text { transtornos } \\
\text { esquizotípicos e } \\
\text { transtornos delirantes }\end{array}$ & $30(18,7)$ & \\
\hline $\begin{array}{l}\text { Transtornos do humor e } \\
\text { afetivos }\end{array}$ & $83(51,9)$ & \\
\hline $\begin{array}{l}\text { Transtornos neuróticos, } \\
\text { transtornos relacionados } \\
\text { com o estresse e } \\
\text { somatoformes }\end{array}$ & $21(13,1)$ & \\
\hline Outras categorias & $26(16,3)$ & \\
\hline $\begin{array}{l}\text { Idade do início do transtorno } \\
\text { psiquiátrico }\end{array}$ & & $26,34(15,29)$ \\
\hline Menos de 18 anos & $52(32,5)$ & \\
\hline De 18 a 40 anos & $77(48,1)$ & \\
\hline Mais de 40 anos & $31(19,4)$ & \\
\hline $\begin{array}{l}\text { Duração do transtorno } \\
\text { psiquiátrico }\end{array}$ & & $15,48(12,04)$ \\
\hline Menos de 5 anos & $30(18,7)$ & \\
\hline De 5 a 15 anos & $64(40,0)$ & \\
\hline Mais de 15 anos & $66(41,3)$ & \\
\hline $\begin{array}{l}\text { Duração do tratamento } \\
\text { psiquiátrico }\end{array}$ & & $8,77(8,02)$ \\
\hline Menos de 5 anos & $64(40,0)$ & \\
\hline De 5 a 15 anos & $70(43,7)$ & \\
\hline Mais de 15 anos & $26(16,3)$ & \\
\hline Crises no último ano & & $3,49(6,51)$ \\
\hline Não teve crises & $66(41,3)$ & \\
\hline Teve crises & $94(58,7)$ & \\
\hline Internações & & $1,49(3,61)$ \\
\hline Nunca foi internado & $94(58,7)$ & \\
\hline Foi internado & $66(41,3)$ & \\
\hline
\end{tabular}

A maioria dos familiares dos pacientes era do sexo feminino $(55,6 \%)$, com mais de 30 anos de idade (84,4\%), escolaridade de ensino fundamental $(51,2 \%)$ e vivia com companheiro (73,1\%). Embora somente $51,2 \%$ dos familiares trabalhassem, 
76,9\% deles possuíam renda própria e tinham como principal fonte de renda o emprego ou trabalho regular (34,4\%), seguida de aposentadoria ou pensão (33,8\%). Quanto ao grau de parentesco do familiar com o paciente, a maioria era representada pelo cônjuge $(43,8 \%)$, seguida por filho $(21,9 \%)$, mãe ou pai $(18,7 \%)$, irmão $(8,7 \%)$, e outros $(6,9 \%)$.

Na tabela 3, são apresentados os resultados dos escores de satisfação dos pacientes e familiares por subescalas e escala global. A média da escala global dos pacientes foi de 4,15. Esse resultado indica satisfação dos pacientes com o serviço. A satisfação para a subescala condições físicas e conforto do serviço apresentou a menor média $(3,48)$, a média de satisfação da subescala competência e compreensão da equipe foi de 4,25, e de 4,33 para subescala acolhida da equipe e ajuda recebida. Para os familiares, a média da escala global foi de 4,24, indicando que, em geral, os familiares dos pacientes estavam satisfeitos com o serviço. Os escores médios de satisfação foram elevados também para as subescalas, sendo 4,25 para a subescala resultados com o tratamento, 4,04 para a subescala acolhida e competência da equipe, e 4,50 para a subescala privacidade e confidencialidade.

Tabela 3. Média de satisfação dos pacientes e familiares atendidos no ambulatório do Hospital de Saúde Mental do Acre por subescalas e escala global, Rio Branco, Acre, 2012/2013

\begin{tabular}{lll}
\hline & Subescalas e escala global & Média (DP) \\
\hline Pacientes & & \\
& 1. Satisfação com a competência e compreensão da equipe & $4,25(0,69)$ \\
& 2. Satisfação com a acolhida da equipe e ajuda recebida & $4,33(0,80)$ \\
& 3. Satisfação com as condições físicas e conforto do serviço & $3,48(0,92)$ \\
& Escala Global & $4,15(0,64)$ \\
Familiares & & \\
& 1. Satisfação com os resultados do tratamento & $4,25(0,85)$ \\
& 2. Satisfação com a acolhida e competência da equipe & $4,04(0,81)$ \\
& 3. Satisfação com a privacidade e confidencialidade & $4,50(0,80)$ \\
& Escala Global & $4,24(0,66)$ \\
\hline
\end{tabular}

Os principais fatores que colaboraram para um alto nível de satisfação dos pacientes e familiares foram: o atendimento da equipe, o sistema de consulta por agendamento e a dispensação de medicamentos.

Embora os pacientes e familiares estejam satisfeitos, $71,3 \%$ e $74,4 \%$, respectivamente, consideram que o serviço precisa ser melhorado. E os principais aspectos apontados foram: infraestrutura, conforto e aparência do serviço, falta de medicamentos, tempo de espera para consulta e número insuficiente de profissionais.

Na análise das variáveis sociodemográficas e clínicas dos pacientes relacionadas à satisfação dos pacientes (Tabela 4), apenas as variáveis idade, idade do início do transtorno psiquiátrico, duração do transtorno psiquiátrico e crise no último ano apresentaram diferença com significância esta- tística entre suas categorias. Dessa forma, os pacientes mais velhos estavam globalmente mais satisfeitos, assim como foi observado maior nível de satisfação naqueles que não tiveram crises no último ano. Os pacientes que tiveram início do transtorno psiquiátrico acima dos 40 anos e os que a duração do transtorno psiquiátrico já tinha mais de 15 anos estavam mais satisfeitos que os demais pacientes.

Tabela 4. Média de satisfação global com o serviço por variáveis sociodemográficas e clínicas dos pacientes atendidos no ambulatório do Hospital de Saúde Mental do Acre, Rio Branco, Acre, 2012/2013

\begin{tabular}{|c|c|c|c|}
\hline Variáveis & $\mathrm{n}$ & Média(DP) & p-valor \\
\hline Sexo & & & 0,86 \\
\hline Masculino & 44 & $4,18(0,59)$ & \\
\hline Feminino & 116 & $4,13(0,65)$ & \\
\hline Idade & & & 0,00 \\
\hline 18 a 29 anos & 24 & $3,75(0,84)$ & \\
\hline 30 a 49 anos & 94 & $4,13(0,58)$ & \\
\hline 50 anos ou mais & 42 & $4,39(0,50)$ & \\
\hline Escolaridade & & & 0,10 \\
\hline Analfabeto & 26 & $4,34(0,54)$ & \\
\hline Ensino Fundamental & 76 & $4,19(0,59)$ & \\
\hline Ensino Médio & 46 & $4,05(0,66)$ & \\
\hline Ensino Superior & 12 & $3,76(0,88)$ & \\
\hline Situação conjugal & & & 0,74 \\
\hline Com companheiro & 89 & $4,16(0,63)$ & \\
\hline Sem companheiro & 71 & $4,12(0,65)$ & \\
\hline Situação ocupacional & & & 0,32 \\
\hline Trabalha & 23 & $4,04(0,64)$ & \\
\hline Não trabalha & 137 & $4,16(0,64)$ & \\
\hline Possui renda própria & & & 0,06 \\
\hline Sim & 109 & $4,22(0,58)$ & \\
\hline Não & 51 & $3,98(0,73)$ & \\
\hline Renda (em salários-mínimos) & & & 0,28 \\
\hline Menor que 1 & 16 & $4,03(0,67)$ & \\
\hline $1 \mathrm{a} 2$ & 87 & $4,26(0,53)$ & \\
\hline 2,1 a 3 & 3 & $4,07(1,07)$ & \\
\hline Maior que 3 & 3 & $4,12(1,04)$ & \\
\hline Não possui renda & 51 & $3,98(0,72)$ & \\
\hline Origem da renda & & & 0,07 \\
\hline Emprego ou trabalho regular & 13 & $3,93(0,68)$ & \\
\hline Aposentadoria ou pensão & 36 & $4,36(0,52)$ & \\
\hline Auxílio do governo & 60 & $4,19(0,57)$ & \\
\hline Categoria diagnóstica & & & 0,07 \\
\hline $\begin{array}{l}\text { Esquizofrenia, transtornos esquizotípicos } \\
\text { e transtornos } \\
\text { delirantes }\end{array}$ & 30 & $4,17(0,60)$ & \\
\hline Transtornos do humor e afetivos & 83 & $4,14(0,64)$ & \\
\hline $\begin{array}{l}\text { Transtornos neuróticos, } \\
\text { transtornos relacionados com o estresse } \\
\text { e somatoformes }\end{array}$ & 21 & $3,84(0,69)$ & \\
\hline Outras categorias & 26 & $4,35(0,56)$ & \\
\hline
\end{tabular}


Tabela 4. Média de satisfação global com o serviço por variáveis sociodemográficas e clínicas dos pacientes atendidos no ambulatório do Hospital de Saúde Mental do Acre, Rio Branco, Acre, 2012/2013

\begin{tabular}{lccc}
\hline Variáveis & $\mathbf{n}$ & Média (DP) & p-valor \\
\hline Idade do início do transtorno psiquiátrico & & & 0,04 \\
Menos de 18 anos & 52 & $4,10(0,65)$ & \\
De 18 a 40 anos & 77 & $4,07(0,64)$ & \\
Mais de 40 anos & 31 & $4,38(0,56)$ & \\
Duração do transtorno psiquiátrico & & & 0,04 \\
Menos de 5 anos & 30 & $4,10(0,75)$ & \\
5 a 15 anos & 64 & $3,99(0,68)$ & \\
Mais de 15 anos & 66 & $4,30(0,49)$ & 0,50 \\
Duração do tratamento psiquiátrico & & & \\
Menos de 5 anos & 64 & $4,11(0,75)$ & \\
5 a 15 anos & 70 & $4,11(0,60)$ & \\
Mais de 15 anos & 26 & $4,32(0,64)$ & \\
Crises no último ano & & & 0,01 \\
Não teve crises & 66 & $4,27(0,62)$ & \\
Teve crises & 94 & $4,05(0,64)$ & \\
Internações & & & 0,09 \\
Nunca foi internado & 94 & $4,20(0,63)$ & \\
Foi internado & 66 & $4,06(0,65)$ & \\
\hline
\end{tabular}

Na análise das variáveis sociodemográficas dos familiares relacionadas à satisfação dos familiares (Tabela 5), somente a variável idade apresentou diferença com significância estatística entre suas categorias, demonstrando que os familiares mais jovens estavam mais satisfeitos com o serviço.

Tabela 5. Média de satisfação global com o serviço por variáveis sociodemográficas dos familiares de pacientes atendidos no ambulatório do Hospital de Saúde Mental do Acre, Rio Branco, Acre, 2012/2013

\begin{tabular}{lccc}
\hline Variáveis & $\mathbf{n}$ & Média (DP) & p-valor \\
\hline Sexo & & & 0,25 \\
Masculino & 71 & $4,14(0,73)$ & \\
$\quad$ Feminino & 89 & $4,30(0,59)$ & \\
Idade & & & 0,03 \\
18 a 29 anos & 25 & $4,37(0,63)$ & \\
30 a 49 anos & 74 & $4,09(0,69)$ & \\
50 anos ou mais & 61 & $4,35(0,59)$ & \\
Escolaridade & & & 0,64 \\
Analfabeto & 14 & $4,29(0,79)$ & \\
Ensino Fundamental & 82 & $4,18(0,68)$ & \\
Ensino Médio & 55 & $4,28(0,55)$ & \\
$\quad$ Ensino Superior & 9 & $4,30(0,89)$ & \\
Situação conjugal & & & 0,88 \\
Com companheiro & 117 & $4,23(0,68)$ & \\
Sem companheiro & 43 & $4,24(0,62)$ & \\
\hline & & & continuação
\end{tabular}

Tabela 5. Média de satisfação global com o serviço por variáveis sociodemográficas dos familiares de pacientes atendidos no ambulatório do Hospital de Saúde Mental do Acre, Rio Branco, Acre, 2012/2013

\begin{tabular}{|c|c|c|c|}
\hline Variáveis & $n$ & Média (DP) & p-valor \\
\hline Situação ocupacional & & & 0,77 \\
\hline Trabalha & 82 & $4,24(0,69)$ & \\
\hline Não trabalha & 78 & $4,23(0,63)$ & \\
\hline Possui renda própria & & & 0,33 \\
\hline Sim & 123 & $4,25(0,67)$ & \\
\hline Não & 37 & $4,17(0,61)$ & \\
\hline Renda (em salários-mínimos) & & & 0,18 \\
\hline Menor que 1 & 17 & $4,32(0,71)$ & \\
\hline 1 a 2 & 89 & $4,32(0,61)$ & \\
\hline 2,1 a 3 & 9 & $4,00(0,67)$ & \\
\hline Maior que 3 & 8 & $4,01(0,94)$ & \\
\hline Não possui renda & 37 & $4,09(0,67)$ & \\
\hline Origem da renda & & & 0,59 \\
\hline Emprego ou trabalho regular & 55 & $4,30(0,64)$ & \\
\hline Aposentadoria ou pensão & 54 & $4,29(0,68)$ & \\
\hline Auxílio do governo & 14 & $4,14(0,63)$ & \\
\hline Parentesco com o paciente & & & 0,77 \\
\hline Mãe ou pai & 30 & $4,21(0,56)$ & \\
\hline Irmão(a) & 14 & $4,24(0,65)$ & \\
\hline Cônjuge & 70 & $4,18(0,75)$ & \\
\hline Filho(a) & 35 & $4,38(0,51)$ & \\
\hline Outro & 11 & $4,18(0,77)$ & \\
\hline
\end{tabular}

\section{DISCUSSÃO}

Os resultados do estudo revelaram que os pacientes e familiares estão satisfeitos com o serviço de saúde mental. Esse resultado está em consonância com os outros estudos brasileiros que avaliaram a satisfação dos pacientes e familiares mediante ao questionário SATIS-BR 3,5,10,12-15. Do mesmo modo, assemelham-se a outros estudos internacionais que evidenciaram níveis elevados de satisfação de pacientes e familiares com os serviços de saúde mental ${ }^{16-21}$.

Neste estudo, a maioria dos pacientes era do sexo feminino, semelhante ao encontrado em estudos brasileiros que avaliaram a satisfação dos pacientes 3,12,13,15. A predominância de pacientes com nível de escolaridade de ensino fundamental e renda de um a dois salários-mínimos sugere o processo de exclusão ainda existente e a dificuldade de reinserção social dos portadores de transtornos mentais, o que consequentemente resulta na carência de oportunidade de emprego, explicando a maioria de os pacientes não trabaIhar e depender financeiramente de auxílio do governo, de aposentadoria ou de pensão. Costa et al. ${ }^{22}$ apontaram essa problemática ao relatarem a ausência de relacionamentos 
extrafamiliares dos pacientes e consideraram essencial o desenvolvimento de ações que favoreçam uma maior integração social do paciente.

Os familiares também apresentaram um perfil sociodemográfico com predominância do sexo feminino, idade de 30 a 49 anos, e escolaridade de ensino fundamental. Esses resultados assemelham-se aos encontrados nos estudos nacionais que avaliaram a satisfação de familiares 5,10,12.

Em relação ao grau de parentesco dos familiares com os pacientes, os cônjuges foram predominantes diferindo de estudos nacionais $5^{5,12}$ e internacionais ${ }^{19,21}$, em que os pais eram os principais cuidadores dos pacientes. Essa divergência pode ser explicada devido à diferença dos diagnósticos dos pacientes estudados, uma vez que nos outros estudos os pacientes eram em sua totalidade ou majoritariamente da categoria diagnóstica de esquizofrenia, transtornos esquizotípicos e transtornos delirantes, enquanto neste estudo a maioria dos pacientes possuía diagnóstico na categoria de transtornos do humor e afetivos. Segundo Terzian et al. ${ }^{23}$, pacientes com esquizofrenia mostraram menores taxas de casamento quando comparados com taxas populacionais. Dessa forma, esses pacientes ficam comumente sob os cuidados de seus pais, uma vez que as características da doença implicam consequências que se refletem em diversos aspectos da vida do paciente, inclusive na vida afetiva e social ${ }^{24}$.

O novo sistema de acesso ao serviço por meio de consultas pré-agendadas foi referido pelos pacientes e familiares como uma mudança positiva no serviço, pois antes eles precisavam chegar de madrugada e por vezes até dormir na fila para conseguir uma consulta. Além disso, a isenção de custos das consultas e a distribuição dos medicamentos também foram referidas como motivos de satisfação com o serviço. Esse fato pode ter contribuído para os altos níveis de satisfação dos pacientes e familiares em relação à escala global, uma vez que fatores como acessibilidade ao serviço e custos com o tratamento estão entre as variáveis determinantes na definição e avaliação da qualidade dos serviços ${ }^{25}$.

Avaliando ainda os resultados positivos das escalas globais, a baixa expectativa desses pacientes e familiares em relação aos serviços deve ser considerada, haja vista que a maioria dos pacientes e familiares possuía um baixo nível de escolaridade, o que pode ter reduzido a capacidade crítica para avaliar com mais objetividade suas percepções quanto ao serviço que lhes é prestado ${ }^{25}$. Além disso, os níveis de satisfação observados não podem ser atribuídos a uma ótima qualidade do serviço, já que altos níveis de satisfação têm sido encontrados nos estudos mesmo quando as expectativas em relação ao serviço são negativas ${ }^{26}$.

Em relação às subescalas, a satisfação dos pacientes foi mais elevada para as características do atendimento da equipe e da ajuda recebida do que para as condições físi- cas e conforto do serviço. Esse fato é constatado na avaliação positiva dos pacientes quanto ao tipo de atendimento que era prestado pela equipe. A maioria considerou que obteve muita ajuda do serviço, visto que sempre era tratado com respeito e dignidade, bem como a acolhida da equipe era muito amigável, e reconhecia a competência do seu terapeuta principal. No entanto, as condições físicas e o conforto do serviço eram sempre referenciados com menor satisfação. Isso corrobora com os resultados dos estudos em que os pacientes apresentaram menores níveis de satisfação em relação ao conforto e à aparência e às condições gerais das instalações ${ }^{3,12,15}$ e consideraram que o serviço precisa ser melhorado, principalmente em relação à estrutura física ${ }^{3,14}$.

Semelhantemente aos pacientes, os familiares estão menos satisfeitos em relação à infraestrutura, ao conforto e à aparência do serviço. Esses fatores foram os mais relatados por eles ao serem questionados sobre os aspectos que precisariam ser melhorados no serviço. Perreault et al. ${ }^{27}$ também verificaram que os itens relacionados à organização dos serviços obtiveram pontuações mais baixas de satisfação dos familiares. Da mesma forma, Gigantesco et al. ${ }^{17}$, ao pesquisarem a satisfação de pacientes e familiares em Roma-Itália, encontraram para o ambiente físico os níveis mais baixos de satisfação. Pena et al. ${ }^{28}$ afirmaram que aspectos relacionados a aparência física das instalações, equipamentos e quadro de profissionais estão entre os principais critérios utilizados pelos usuários para avaliar os serviço de saúde.

Os resultados das subescalas para os familiares apresentaram satisfação, principalmente, em relação às medidas tomadas para assegurar a privacidade e confidencialidade no serviço. Conquanto, um menor nível de satisfação dos familiares foi demonstrado em relação à acolhida e à competência da equipe, e aos resultados do tratamento. Esse resultado reflete o baixo contato dos familiares com a equipe do serviço, revelando um distanciamento do familiar em relação ao tratamento do paciente, em que alguns familiares demonstraram incerteza na compreensão da equipe sobre o problema do paciente e ajuda que este precisava, assim como se o serviço que o paciente obtinha era o que ele necessitava.

Dessa forma, a dificuldade no contato com a equipe se traduz em uma menor satisfação com os resultados do tratamento. Haja vista que, diante das dificuldades de relacionamentos com a equipe, os familiares passam a avaliar com menor potencial a ajuda do serviço em relação às necessidades do paciente. Esses dados indicam a importância da relação entre a família e a equipe para a satisfação com o tratamento, a qual necessita de esforços dirigidos a uma maior participação e parceria. Corroborando esse resultado, Perreault et al. ${ }^{27}$ verificaram que a colaboração entre familiares e profissionais foi o mais importante preditor da satisfação dos familiares com os serviços de saúde. 
Neste estudo, a maior idade esteve relacionada com uma maior satisfação dos pacientes, consoante ao estudo de Silva et al. ${ }^{15}$, que, ao estudarem 110 pacientes de três instituições públicas de saúde mental, encontraram a maior idade como segunda variável preditora de satisfação dos pacientes com os serviços. Esse mesmo resultado foi encontrado por Rahmqvist e Bara ${ }^{29}$, na Suécia, ao avaliarem a satisfação de 7.245 usuários. Silva et al. ${ }^{15}$ explicaram essa relação em consequência da diminuição da expectativa que as pessoas idosas devem ter com o serviço se declarando assim mais satisfeitas.

A variável crise, no último ano, também demonstrou relação com a satisfação dos pacientes. Os pacientes os quais não tiveram crises estavam mais satisfeitos com o serviço do que os que tiveram. A redução das crises indica para os pacientes que o tratamento oferecido pelo serviço está apresentando resultados positivos ${ }^{3}$.

Para os familiares, apenas a variável idade esteve relacionada com o nível de satisfação. Os familiares mais jovens estavam mais satisfeitos com o serviço. No entanto, Bandeira et al..$^{5}$ não observaram nenhuma variável sociodemográfica dos familiares associada à satisfação com os serviços de saúde mental.

O presente estudo tem como limitação a impossibilidade de generalização dos seus resultados, uma vez que a amostra não foi selecionada aleatoriamente da população-alvo. O fato de terem sido selecionados apenas pares de pacientes e familiares também se constitui uma limitação do estudo, pois aqueles que não responderam ao questionário poderiam ter diferentes opiniões em relação aos que participaram do estudo, principalmente pelo fato de que pacientes os quais são acompanhados por familiares podem ter um nível de satisfação maior, já que desfrutam de suporte familiar.

\section{CONCLUSÕES}

Os resultados deste estudo indicaram que os pacientes e familiares estão satisfeitos com o serviço de saúde mental. Esse resultado traduz a visão positiva que esses usuários têm do serviço ao qual estão integrados.

Este estudo identificou que a maior idade esteve relacionada com maior satisfação dos pacientes e que os pacientes que não tiveram crises estavam mais satisfeitos com o serviço do que os que tiveram, assim como os familiares mais jovens também tinham maior nível de satisfação.

Os resultados apontam para necessidades de melhorias no serviço no que diz respeito aos aspectos relacionados à infraestrutura, ao conforto e à aparência dos serviços. Dessa forma, verifica-se a importância de maiores investimentos nessas áreas, pois essas carências podem comprometer o trabalho das equipes e os seus resultados. Além de que, reconhecendo os usuários dos serviços como cidadãos de direitos, eles devem usufruir de condições adequadas de in- fraestrutura, de modo que favoreça a qualidade da assistência de saúde que lhe é prestada.

Este estudo ressalta a necessidade de criação de estratégias no serviço que favoreçam uma maior participação do familiar no tratamento do usuário. E destaca, principalmente, a pertinência de avaliar as perspectivas de pacientes e familiares, a fim de promover maior envolvimento destes no planejamento, na implementação e na avaliação dos serviços de saúde mental.

As pesquisas de satisfação por meio da utilização de escalas são importantes indicadores da qualidade dos serviços, e seus resultados permitem a identificação de fatores que contribuem para a satisfação dos pacientes e familiares com o serviço. No entanto, o uso desses dados deve ser incrementado por uma avaliação mais profunda dos pontos fortes e das necessidades de melhorias do serviço, inclusive por meio de pesquisas de abordagem qualitativa. Estudos futuros poderão, também, esclarecer a relação entre a satisfação e a adesão ao tratamento psiquiátrico.

\section{CONTRIBUIÇÕES INDIVIDUAIS}

Todos os autores contribuíram significativamente na concepção e no desenho do estudo, na análise e na interpretação dos dados, na elaboração do artigo e na revisão do conteúdo.

\section{CONFLITOS DE INTERESSE}

Priscila Oliveira de Miranda, Orivaldo Florêncio de Souza e Terezinha de Freitas Ferreira não possuem conflitos de interesse a serem declarados.

\section{REFERÊNCIAS}

1. Organização Mundial de Saúde (OMS). Relatório sobre a saúde no mundo 2001: Saúde mental: nova concepção, nova esperança. Genebra: OMS/OPAS, 2001.

2. Bandeira M, Calzavara MGP, Costa CS, Cesari L. Avaliação de serviços de saúde mental: adaptação transcultural de uma medida da percepção dos usuários sobre os resultados do tratamento. J Bras Psiquiatr. 2009;58(2):107-14.

3. Kantorski LP, Jardim VR, Wetzel C, Olschowsky A, Schneider JF, Heck RM, et al. Satisfação dos usuários dos centros de atenção psicossocial da região Sul do Brasil. Rev Saude Publica. 2009:43 Suppl 1:29-35.

4. Coimbra VCC, Kantorski LP, Oliveira MM, Pereira DB, Nunes CK, Eslabão AD. Avaliação da satisfação dos usuários com o cuidado da saúde mental na Estratégia Saúde da Família. Rev Esc Enferm USP. 2011;45(5):1150-6.

5. Bandeira M, Silva MA, Camilo CA, Felício CM. Satisfação de familiares de pacientes psiquiátricos com os serviços de saúde mental e seus fatores associados. J Bras Psiquiatr. 2011;60(4):284-93.

6. Bandeira M, Pitta AMF, Mercier C. Escalas da OMS de avaliação da satisfação e da sobrecarga em serviços de saúde mental: qualidades psicométricas da versão brasileira. I Bras Psiquiatr. 1999;48(6):233-44. 
7. Machin D, Campbell MJ, Fayers PM, Pinol APY. Sample size tables for clinical studies. OXford: Blackwell Science Ltd.; 1997.

8. Bandeira M, Pitta AMF, Mercier C. Escala de avaliação da satisfação dos usuários em serviços de saúde mental: SATIS-BR. J Bras Psiquiatr. 2000a;49(8):293-300.

9. Bandeira M, Pitta AMF, Mercier C. Escalas brasileiras de avaliação da satisfação (SATISBR) e da sobrecarga (IMPACT0-BR) da equipe técnica em serviços de saúde mental. J Bras Psiquiatr. 2000b;49(4):105-15.

10. Bandeira M, Mercier C, Perreault M, Libério MMA, Pitta AMF. Escala de Avaliação da Satisfação dos Familiares com Serviços de Saúde Mental: SATIS-Br. J Bras Psiquiatr. 2002;51(3):153-66.

11. Bandeira M, Silva MA. Escala de Satisfação dos Pacientes com os Serviços de Saúde Mental (SATIS-BR): estudo de validação. J Bras Psiquiatr. 2012;61(3):124-32.

12. Camilo CA, Bandeira M, Leal RMAC, Scalon JD. Avaliação da satisfação e sobrecarga em um serviço de saúde mental. Cad Saude Colet. 2012;20(1):82-92.

13. Heckert U, Teixeira LS, Trindade AS. Avaliação da satisfação dos usuários do Centro Regional de Referência em Saúde Mental (CRRESAM) da região central de Juiz de Fora, MG. HU Rev. 2006;32(1):15-9.

14. Jaegger RC, Guitton AP, Lyrio JM, Santos MM, Freitas RCO, Gonçalves SR, et al. A experiência de morar fora: avaliação da satisfação de usuários em um serviço de saúde mental. Cad Saude Colet. 2004;12(1):27-39.

15. Silva MA, Bandeira M, Scalon JD, Quaglia MAC. Satisfação dos pacientes com os serviços de saúde mental: a percepção de mudanças como preditora. J Bras Psiquiatr. 2012;61(2):64-71.

16. Gani N, Saeed K, Minhas FA, Anjuman N, Waleed M, Fatima G. Assessment of patient satisfaction with mental health services in a tertiary care setting. J Ayub Med Coll Abbottabad. 2011;23(1):43-6.

17. Gigantesco A, Picardi A, Chiaia E, Balbi A, Morosini P. Patients' and relatives' satisfaction with psychiatric services in a large catchment area in Rome. Eur Psychiatry. 2012;17(3):139-47.
18. Martin JS, Petr CG, Kapp SA. Consumer satisfaction with children's mental health services. Child Adolesc Social Work J. 2003;20(03):211-26.

19. Stengard E, Honkonen T, Koivisto AM, Salokangas RKR. Satisfaction of caregivers of patients with schizophrenia in Finland. Psychiatr Serv. 2000;51(8):1034-9.

20. Svensson B, Hansson L. Satisfaction with mental health services. A user participation approach. Nord J Psychiatry. 2006;60(5):365-71.

21. Urízar AC, Maldonado JG. Satisfaction with mental health services in a Latin American community of carers of patients with schizophrenia. Community Ment Health J. 2009:45(4):285-9.

22. Costa CS, Bandeira M, Cavalcanti RLA, Scalon JD. A percepção de pacientes e familiares sobre os resultados do tratamento em serviços de saúde mental. Cad Saude Publica. 2011;27(5):995-1007.

23. Terzian ACC, Andreoli SB, Razzouk D, Chaves AC, Mari J. Fertility and fecundity of an outpatient sample with schizophrenia. Rev Bras Psiquiatr. 2006;28(4):305-7.

24. Oliveira RM, Facina PCBR, Junior ACS. A realidade do viver com esquizofrenia. Rev Bras Enferm. 2012;65(2):309-16.

25. Vaitsman J, Andrade GRB. Satisfação e responsividade: formas de medir a qualidade e a humanização da assistência à saúde. Cien Saude Colet. 2005;10(3):599-613.

26. Esperidião M, Trad LAB. Avaliação de satisfação de usuários. Cien Saude Colet. 2005;10:303-12.

27. Perreault M, Rousseau M, Provencher H, Roberts S, Milton D. Predictors of caregiver satisfaction with mental health services. Community Ment Health J. 2012;48(2):232-7.

28. Pena MM, Silva EMS, Tronchin DMR, Melleiro MM. 0 emprego do modelo de qualidade de Parasuraman, Zeithaml e Berry em serviços de saúde. Rev Esc Enferm USP. 2013:47(5):1235-40

29. Rahmqvist M, Bara AC. Patient characteristics and quality dimensions related to patient satisfaction. Int I Qual Health Care. 2010;22(2):86-92. 